cells (DC) and/or the transformation of synoviocytes to follicular DC may play a key role in this process. In our model a so-called amplifier gene stimulates this chemo-attraction of and/or the differentiation to DC's. The DC's present self-peptides not to disease-inducing but to regulatory auto-reactive $\mathrm{T}$ cells that have been recruited also to the inflamed synovium. These regulatory T cells recognise self-peptides such as DERAA peptides from the HV III region of certain HLA-DR alleles presented by DR or DQ molecules. This DC-regulatory T cell interaction is an essential checkpoint for the control of the inflammation and is controlled by HLA class II genes. In the absence of protective HLA alleles and therefore self-reactive regulatory $\mathrm{T}$ cells this checkpoint fails and this results in a local loss of tolerance and a (severe) chronic arthritis (RA).

This model has several important implications for the immunotherapy of RA:

- We may stop the search for a unique target antigen for disease-inducing $\mathrm{T}$ cells

- Immunotherapy that is exclusively targeted at $\mathrm{T}$ cells may have an adverse effect

- Immunotherapy strategies for RA should either be targeted at the re-establishment of a normal phenotype in synoviocytes or on restoring a functional immunoregulatory loop.

\section{SP0051 HSP REACTIVE T CELLS AS INFLAMMATION REGULATORS}

${ }^{1} W$ Van Eden, ${ }^{1} \mathrm{~L}$ Paul, ${ }^{1} \mathrm{U}$ Wendling, ${ }^{1} \mathrm{P}$ Van Kooten, ${ }^{1} \mathrm{~J}$ Bos-de Ruijter, ${ }^{1} \mathrm{R}$ Van der Zee, ${ }^{2} \mathrm{~B}$ Prakken. ${ }^{1}$ Department of Infectious Diseases and Immunology, Faculty of Veterinary Medicine, Utrecht University, Utrecht, The Netherlands; ${ }^{2}$ Department of Pediatric Immunology, University Hospital for Children and Youth, Utrecht, The Netherlands

\subsection{6/annrheumdis-2001.10}

Immunisation with heat shock proteins has protective effects in models of induced arthritis. Analysis has shown a reduced synovial inflammation in such protected animals. Adoptive transfer and immunisation with selected T cell epitopes (synthetic peptides) have indicated the protection to be mediated by $\mathrm{T}$ cells directed to conserved hsp epitopes. This was shown first for mycobacterial hsp $60^{1}$ and later for mycobacterial hsp $70 .^{2,3}$

Fine specificity analysis showed that such $\mathrm{T}$ cells were crossreactive with the homologous self hsp. Therefore protection by microbial hsp reactive $\mathrm{T}$ cells can be by cross-recognition of self hsp overexpressed in the inflamed tissue. Preimmunisation with hsp leads to a relative expansion of such self hsp cross-responsive $\mathrm{T}$ cells. The regulatory nature of such $\mathrm{T}$ cells may originate from mucosal tolerance maintained by commensal flora derived hsp or from partial activation through recognition of self hsp as a partial agonist (Altered Peptide Ligand) or in the absence of proper costimulation. Recently, we reported the selective upregulation of B7.2 on microbial hsp60 specific T cells in response to self hsp60. ${ }^{4}$ Through a preferred interaction with CTLA-4 on proinflammatory $\mathrm{T}$ cells this may constitute an effector mechanism of regulation. Also, regulatory T cells produced IL10.

\section{REFERENCES}

1 Anderton SM, van der Zee R, Prakken B, Noordzij A, van Eden W. Activation of T cells recognizing self $60-k D$ heat shock protein can protect against experimental arthritis. J Exp Med. 1995;181:943-52

2 Tanaka S, Kimura Y, Mitani A, Yamamoto G, Nishimura H, Spallek R, Singh M, Noguchi T, Yoshikai Y. Activation of T cells recognizing an epitope of heat-shock protein 70 can protect against rat adjuvant arthritis. J Immunol. 1999;163:556065
3 Wendling U, Paul L, van der Zee R, Prakken B, Singh M, van Eden W. A conserved mycobacterial heat shock protein (hsp) 70 sequence prevents adjuvant arthritis upon nasal administration and induces IL-10-producing T cells that crossreact with the mammalian self-hsp70 homologue. J Immunol. 2000;164:2711-17

4 Paul AG, van Der Zee R, Taams LS, van Eden W. A self-hsp60 peptide acts as a partial agonist inducing expression of B7-2 on mycobacterial hsp60-specific T cells: a possible mechanism for inhibitory $\mathrm{T}$ cell regulation of adjuvant arthritis? Int Immunol. 2000;12:1041-50

\section{SP0052 DETECTION OF ANTIGEN-SPECIFIC T CELLS}

P Bowness. MRC Human Immunology Group, IMM, John Radcliffe Hospital, Oxford, UK

10.1136/annrheumdis-2001.11

Antigen-specific $\mathrm{T}$ cells are thought to drive the inflammatory response in a number of rheumatic diseases including RA and the spondyloarthropathies. $T$ cells recognise specific combinations of antigenic peptides bound to of HLA molecules, and subsequently proliferate and perform effector functions including cytokine secretion. Techniques have been developed to detect these T cells (usually ex vivo or in vitro), based upon their antigenic specificity, their function, and their clonality. Some techniques combine these attributes. Recently fluorescent-labelled multimers (usually tetramers) of synthetic HLA/peptide combinations have been used to stain $\mathrm{T}$ cells of defined antigen specificity. The use of HLA /peptide tetramers has greatly increased our understanding of the role of $\mathrm{T}$ cells in viral infections and given some insight into rheumatic diseases. Other techniques have been developed that detect cytokines produced in response to specific antigens (i.e. detecting function), or that detect populations of T cells carrying the same or related T cell receptors. Our ability to detect antigen-specific $\mathrm{T}$ cells has important implications for our understanding of disease pathogenesis, and for monitoring disease progress. These methodologies also offer therapeutic potential.

\section{Polymyalgia rheumatica - Thursday 14 June, 16.00-17.30/Small Hall}

\section{SP0053 EUROPEAN DIAGNOSTIC CRITERIA FOR POLYMYALGIA RHEUMATICA}

H Bird. Clinical Pharmacology Unit, University of Leeds, Leeds, UK

10.1136/annrheumdis-2001.12

A variety of separate diagnostic criteria sets exist for polymyalgia rheumatica. These include those proposed in $1979,{ }^{1} 1981^{2}$ and 1989. ${ }^{3}$ All differ slightly, the optimum criteria still having to be determined.

In 1997, the Polymyalgia Rheumatica Special Interest Group was established under the auspices of the EULAR Standing Committee for International Clinical Studies. Twenty participating centres throughout Europe between them promised to make available 200 new patients each year for the comparison of criteria sets. In parallel patients, where suitable, were recruited to a study to evaluate, for the first time, response criteria for this condition. It was hoped that both studies would lead to more accurate assessment of the several drugs then being claimed to be steroid sparing agents. 
Collection of data terminated at the end of the year 2000 . Although, as this abstract is prepared, some data sets are still awaited, it is likely that analysis will be based on data from 312 patients judged to have polymyalgia rheumatica recruited from 13 centres.

The vast majority of these patients, judged by a consensus of experts to have polymyalgia rheumatica, have provided a database for comparison of the various diagnostic criteria sets.

On the basis of this, optimum European diagnostic criteria will be proposed. Investigators are encouraged to use this in their research on polymyalgia rheumatica to further substantiate their reliability. A handout listing the new criteria will be available at the time of the presentation.

\section{REFERENCES}

1 Bird HA, et al. Ann Rheum Dis. 1979;38:434-9

2 Jones JG, Hazleman BL. Ann Rheum Dis. 1981;40:1-5

3 Nobunaga $\mathrm{M}$, et al. Jpn J Med. 1989:28:452-6

\section{SP0054 POLYMYALGIA RHEUMATICA REMISSION CRITERIA}

B Leeb. Lower Austrian Center for Rheumatology, Stockerau Hospital, Stockerau, Austrialntroduction

\subsection{6/annrheumdis-2001.13}

Polymyalgia rheumatica (PMR) is not a benign disease. Additional morbidity may arise from prolonged administration of high doses of corticosteroids. No stringent response criteria have been established yet, however, they obviously seem to be of great importance for the quality of patient care and to compare alternative treatment modalities against the golden standard of steroid treatment as steroid sparing medications would be most desirable in PMR.

Patients and methods Patients from several European countries were enrolled into a study to validate existing diagnostic criteria for PMR as well as into the remission criteria study. Serological findings (ESR, CRP, haemoglobin, serum iron and alpha2-globuline) and clinical features (VAS pain, VAS physicians assessment, morning stiffness, elevation of upper limbs, myalgia, tenderness on pressure of upper/lower limbs, response time after initiation of steroids, head aches, swelling or tenderness of the temporal artery and masseter muscle claudication) were chosen for the characterisation of disease activity. After the initial examination patients were seen at week $1,4,8,16,24$ and 32, study endpoint was at week 48 . Starting dose and reduction of corticosteroids as well as co-medication was at the discretion of the local investigator. Patients found to have a disease other than PMR will be excluded from final analysis in the response criteria study.

Results As expected most of the patients responded well and quickly to steroid treatment. The results concerning the single response parameters will be given on date. Consisting of those criteria performing best a response score should be proposed. The efficacy of treatment may thus easily be reflected by a single figure.

\section{SP0055 POLYMYALGIA RHEUMATICA NEW TREATMENT (NEW STEROID SPARING AGENTS)}

GG Hunder. Rheumatology Department, Mayo Clinic, Rochester, USA
Polymyalgia rheumatica (PMR) responds well to glucocorticoids such as prednisone when given in small to medium-sized doses. But because the condition tends to persist for months or even years, adverse effects develop in most patients (Gabriel, et al. Arthritis Rheum 1997;40:1873). A variety of steroid sparing drugs have been tried. One NSAID, Tenidap, showed steroid sparing efficacy but also relatively frequent adverse effects. The new Cox-2 inhibitor drugs have not been studied adequately in PMR to determine if they have a role in certain cases.

Experience with cytotoxic drugs has been mainly in cases of giant cell arteritis (GCA), some of whom also had PMR. Even in GCA, cytotoxic investigations have been limited. Because of the close link between GCA and PMR it would be presumed that drugs useful for GCA would also be effective in PMR. Methotrexate has been studied most. Two recent randomised, doubleblind, placebo-controlled studies using methotrexate as a steroid sparing agent are the best work on this question to date, but gave different results. In one (Jover, et al. Ann Intern Med 2001;134:106), 33 patients completed the trial. 18 patients were given prednisone plus placebo, and 15 were given daily prednisone plus $10 \mathrm{mg}$ methotrexate per week. All were followed for 24 months. The group receiving methotrexate with prednisone experienced fewer relapses $(\mathrm{p}=0.02)$ and used significantly less prednisone $(\mathrm{p}=0.004)$ over the study period. The second study (Hoffman, et al. In press) was also a prospective randomised double-blind study. 98 patients were enrolled. 51 received methotrexate (mean dose $15 \mathrm{mg}$ per wk) plus prednisone, and 47 received placebo and prednisone. The results showed that the methotrexate group was not different from the placebo group regarding outcome and prednisone dose. The reasons for the differences between these studies is not completely clear but will be discussed. Thus, at this time there are no steroid sparing drugs that can be relied on for PMR. At this time in the practice setting we would recommend a trial of methotrexate only in patients who at very high risk of developing steroid side effects. Additional investigations with this drug and others is needed.

\section{Clinical epidemiology in practice - Thursday 14 June, 16.00-17.30/Meeting Hall IV}

\section{SP0056 MEASUREMENT ISSUES}

A Tennant. Rheumatology and Rehabilitation Research Unit, University of Leeds, Leeds, UK

10.1136/annrheumdis-2001.15

In a recent European survey of outcome measures for rheumatoid arthritis over 60 different instruments were found to be in use. For low back pain, over 100 different instruments were found to be in use. If, for example, measuring disability is considered important, what are the characteristics of a good measure of disability? How do we chose between competing instruments?

Recent advances in our understanding of outcome measurement have identified a number of fundamental criteria that should be met, prior to more established tests of reliability, validity and responsiveness. The two most important characteristics are unidimensionality and absence of Differential Item Functioning (DIF).

The ICIDH helps us to think conceptually about outcomes, and contributes to measurement by identifying unidimensional 